doi: http://dx.doi.org/10.5892/ruvrv.2012.102.211219

\title{
Análise das prescrições de medicamentos dispensados na Farmácia Escola da UNIFAL-MG
}

\author{
Samir Antonio Rodrigues ABJAUDE ${ }^{1}$ \\ Ariane Cristina Barboza ZANETTI ${ }^{2}$ \\ Luciene Alves Moreira MARQUES ${ }^{3}$ \\ Ricardo Radighieri RASCADO ${ }^{*}$
}

\begin{abstract}
${ }^{1}$ Farmacêutico. Universidade Federal de Alfenas, Minas Gerais. Brasil. E-mail: samirabjaude@ hotmail.com
${ }^{2}$ Mestranda em Ciências pela Escola de Enfermagem de Ribeirão Preto. Universidade de São Paulo, São Paulo. Brasil. E-mail: arianezanetti@hotmail.com

${ }^{3}$ Doutora, Professora de Deontologia e Atenção Farmacêutica. Universidade Federal de Alfenas, Minas Gerais. Brasil. E-mail: lualvesmarques@gmail.com

${ }^{4}$ Doutor Professor de Atenção Farmacêutica da Faculdade de Ciências Farmacêuticas. Universidade Federal de Alfenas, Minas Gerais. Brasil. E-mail: ricardounifal@gmail.com
\end{abstract}

*AUTOR CORRESPONDENTE: Ricardo Radighieri Rascado

Endereço: Universidade Federal de Alfenas, UNIFAL-MG, Campus Alfenas, Rua Gabriel Monteiro da Silva, 700, 37130-000, Alfenas-MG, Brasil

Telefax: (35) 32991349

E-mail: ricardounifal@gmail.com

Recebido em: 19/09/2012 - Aprovado em: 18/12/2012 - Disponibilizado em: 30/12/2012

\section{RESUMO}

A prescrição de medicamentos é essencial no tratamento farmacoterapêutico trazendo informações importantes ao farmacêutico e ao usuário. Portanto, diminuir a ocorrência de erros de prescrição é importante para a promoção do uso racional de medicamentos. Este trabalho analisou prescrições de medicamentos dispensados na Farmácia-Escola da UNIFAL-MG identificando os erros mais freqüentes. Foram analisadas 323 prescrições entre 2006 e 2008. Utilizando a lei 5991/73, foi verificada a presença das seguintes informações: legibilidade; identificação profissional; identificação do paciente; identificação das substâncias; dosagem; forma farmacêutica; quantidade; posologia; endereço e data da consulta e; interações e aspectos farmacológicos. A análise das prescrições revelou que 30,95\% apresentaram ilegibilidade ou dificuldade de leitura; $15,78 \%$ ausência de identificação do prescritor; 6,19\% ausência de dosagem; 4,02\% ausência da forma farmacêutica; 5,57\% descrição da quantidade; $10,52 \%$ de posologia errada/incompleta; 43,34\% ausência de data/endereço; e 3,10\% medicamentos incompatíveis. Este trabalho demonstra a má qualidade das prescrições de medicamentos, indicando um despreparo ou mesmo indiferença dos profissionais prescritores para com o usuário de medicamentos. Faz-se necessário sensibilizar os profissionais de saúde à importância da qualidade da prescrição, para dispensação e administração de medicamentos e promoção do uso racional, proporcionando ao paciente maior eficácia e segurança do seu tratamento farmacológico.

Palavras-chaves: Erros de Medicação. Prescrições de Medicamentos. Medicamentos sob Prescrição. Assistência Farmacêutica. Estudos Retrospectivos.

\begin{abstract}
The prescription of drugs is essential in the pharmacotherapeutic treatment bringing important information to the pharmacy and the user. Therefore reduce the occurrence of prescription errors is important for promoting the rational use of medicines. This study analyzed drug prescriptions dispensed in the Pharmacy School of UNIFAL-MG identifying the most frequent mistakes. We analyzed 323 prescriptions between 2006 and 2008. Using the law 5991/73, there was the presence of the following information: readability; professional identification, patient identification, identification of substances, dosage, dosage form, quantity, address and date of consultation; and interactions and pharmacological aspects. Analysis of requirements showed that $30.95 \%$ were illegible or difficult to read; $15.78 \%$ failure to identify the prescriber; $6.19 \%$ lack of assay $4.02 \%$ absence of the dosage form; $5.57 \%$ description of quantity; $10.52 \%$ dosage wrong / incomplete; $43.34 \%$ lack of data / address, and $3.10 \%$ drugs incompatible. This study demonstrates the poor quality of drug prescriptions, indicating a lack of preparation or even indifference toward the prescribers of drug users. It is necessary to sensitize health professionals to the importance of quality of prescribing to dispensing and administration of medicines and promoting rational use, providing the patient with greater efficacy and safety of its pharmacological treatment.
\end{abstract}

Keywords: Medication Errors. Drug Prescriptions. Prescription Drugs. Pharmaceutical Care. Retrospective Studies. 


\section{INTRODUÇÃO}

A prescrição de medicamento indica o resultado da perspicácia diagnóstica e da competência terapêutica do médico, sendo uma relação entre o profissional habilitado e o paciente, fornecendo instruções destinadas ao alívio ou cura de uma enfermidade. É um instrumento essencial para o tratamento e para o Uso Racional de Medicamentos, pois deve conter as informações indispensáveis sobre o medicamento (FARIAS et al., 2007).

A prescrição médica é um documento escrito que indica o resultado do raciocínio clínico formado com os dados antecedentes e as informações do exame físico do paciente, e se necessário, de dados de exames, permitindo a avaliação da qualidade do atendimento, causando uma contribuição para a otimização do quadro clínico e dos recursos propostos ao cuidado da saúde (MEINERS, BERGSTEN-MENDES, 2001).

Uma boa prescrição deve conter informações suficientes que permitam ao farmacêutico uma análise correta sobre a indicação, posologia e possíveis erros no momento da dispensação do medicamento. Desta forma, a prescrição precisa ser padronizada, seguindo a legislação de cada país.

No Brasil a normatização da prescrição deve ser seguida de acordo com Lei 5991/73, que determina as informações mínimas que devem estar presentes nas prescrições: nome do paciente, data e endereço da clínica ou consultório, nome do medicamento, concentração, dosagem, posologia, forma farmacêutica, via de administração, frequência, horário de administração, assinatura e registro no Conselho do profissional prescritor, e ainda devem ser legíveis e sem rasuras. Em relação ao nome do medicamento no âmbito do Sistema Único de Saúde (SUS), deve ser adotado obrigatoriamente a Denominação Comum Brasileira (DCB) e na sua ausência a Denominação Comum Internacional (DCI).

“A prescrição de um medicamento é considerada racional, sempre que o tratamento farmacológico seja de fato indicado; o medicamento prescrito seja eficaz para o quadro clínico do paciente; o medicamento seja utilizado na dose correta e por período de tempo apropriado; seja a alternativa farmacoterapêutica mais segura e, preferencialmente, de menor custo" (COLOMBO et al., 2004).

No entanto, a prescrição médica pode resultar em consequências maléficas para o paciente e contribuir significativamente para o índice total de erros de medicação, pois é um ato complexo e sujeito a diversos erros. Esse problema se eleva na proporção em que os profissionais não conseguem entender a prescrição devido à letra ilegível ou a ausência de informações indispensáveis para a adequada dispensação e orientação, como via de administração, frequência de uso, dentre outros (FREIRE et al., 2004). 
Além da dificuldade da leitura do nome dos medicamentos, há também situações complicadas como a ilegibilidade da assinatura do profissional prescritor ou ausência de identificação deste, pois os profissionais responsáveis pela dispensação, como o farmacêutico, não sabem a quem contatar para esclarecer possíveis dúvidas.

As responsabilidades do profissional farmacêutico destacam-se perante as prescrições medicamentosas. Este profissional da saúde encontra-se envolvido na interface entre a dispensação de medicamentos e a sua administração pelo paciente, podendo ser considerado uma peça-chave na segurança da qualidade do tratamento médico (RUPP et al., 1992). "Ele representa uma das últimas oportunidades de, ainda dentro do sistema de saúde, identificar, corrigir ou reduzir possíveis riscos associados à terapêutica. Suas responsabilidades, no momento da dispensação, são múltiplas. Envolvem questões de cunho legal, técnico e clínico. No momento que antecede o aviamento da receita/prescrição, o farmacêutico pode examiná-la atentamente, cruzando estas informações com dados da história clínica do paciente. É imprescindível o total entendimento das informações constantes na prescrição" (BROWN, 1997).

$\mathrm{O}$ ato da dispensação tem tal importância, pois além da entrega do medicamento para o paciente, também há dispensação de informações envolvidas para a melhor eficácia do tratamento, não sendo apenas a ocasião em que determinada receita ou prescrição é aviada, e nem ao menos a troca de mercadorias por receitas médicas. A continuidade da comunicação, começado pela consulta médica, tem sequência no estabelecimento farmacêutico, onde o paciente será orientado a respeito dos itens da prescrição médica, dose, posologia, orientados na reconstituição, armazenamento, modo correto de utilização do medicamento, interações medicamentosas, eventos adversos, dentre outros (NEWTON et al. 1996 citado por PEPE, CASTRO, 2000). Esta dispensação, área de conhecimento do farmacêutico, desvalorizada pelas pessoas, é composta por potenciais pacientes, como atividade de elevada importância (ARANCÍBIA, 1990 citado por PEPE, CASTRO, 2000). Normalmente, o paciente resiste em associar o recinto farmacêutico como um dos estabelecimentos privilegiados para sua assistência. Nem ao menos este espera e solicita um contato maior com o farmacêutico do estabelecimento. No entanto, a dispensação pode tornar-se encontro vantajoso entre pacientes e dispensador (JOHSON et al., 1996; NAU et al., 1997; citado por PEPE, CASTRO, 2000).

A conferência da prescrição deve ser realizada pelo farmacêutico, antes da separação dos medicamentos e dúvidas relativas à caligrafia da prescrição devem ser esclarecidas com o prescritor e nunca devem ser deduzidas. 
Distinguindo-se desses estudos, a presente pesquisa focalizou a questão da prescrição do profissional habilitado pela ótica da dispensação farmacêutica. Para tanto,

\section{MATERIAL E MÉTODOS}

Tipo de estudo - Trata-se de estudo descritivo, retrospectivo do tipo quantitativo, envolvendo a determinação de falhas no receituário dos medicamentos.

Local do estudo - $\mathrm{O}$ estudo foi desenvolvido na Farmácia Escola da Universidade Federal de Alfenas-MG (UNIFAL-MG). A seleção desse local devese ao fato de ser um estabelecimento de ensino, onde existe facilidade em analisar as prescrições antes de atender os usuários de medicamentos.

\section{População e Amostra - Foram} coletadas e analisadas as 323 prescrições de medicamentos recebidas na Farmácia Escola da UNIFAL-MG durante o $1^{\circ}$ semestre de 2006 até o $2^{\circ}$ semestre de 2008 . Foi solicitado o consentimento do paciente para que a prescrição fosse scaneada e imediatamente devolvida para o paciente.

\section{Procedimento de Coleta de Dados -}

Foi elaborado um checklist com as

\section{RESULTADOS}

Do total de prescrições analisadas, $30,95 \%$ apresentaram-se ilegíveis ou com dificuldade de leitura; em 15,78\% faltaram assinatura, registro profissional e/ou o carimbo; 0,30\% não apresentaram o nome do procurou-se verificar se as receitas apresentavam ou não informações que podem dificultar ou até inviabilizar a dispensação.

informações que obrigatoriamente devem estar presentes nas prescrições conforme a Lei 5991/73. Assim, a análise dos dados levou em consideração as informações pertinentes na lei, que devem ser: a) legibilidade; b) identificação do profissional (CRM, carimbo e assinatura); c) identificação do paciente; d) identificação das substâncias (DCB); e) dosagem; f) forma farmacêutica; g) quantidade; h) posologia; i) endereço do atendimento e data da consulta; também foi analisada a j) presença de possíveis interações medicamentosas.

Os resultados foram contabilizados e expressos em gráfico como valor absoluto de eventos no total de receitas analisadas.

paciente; $21,05 \%$ das prescrições apresentaram nomes comerciais dos medicamentos; 6,19\% não apresentaram dosagem dos medicamentos; em 4,02\% faltaram a forma farmacêutica; $5,57 \%$ das 
receitas apresentaram falta de quantidade; $10,52 \%$ estavam com posologia errada ou incompleta; 43,34\% não apresentavam o endereço ou a data do atendimento do paciente; e $3,10 \%$ apresentaram incompatibilidade entre os medicamentos. Desta forma $69 \%$ das prescrições continham informações incompletas que dificultaram a dispensação do medicamento e $31 \%$ foram dispensadas embora apresentassem problemas.

A figura 1 sintetiza os resultados apresentados depois da análise de cada receita.

Figura 1 - Distribuição dos dados analisados nas prescrições.

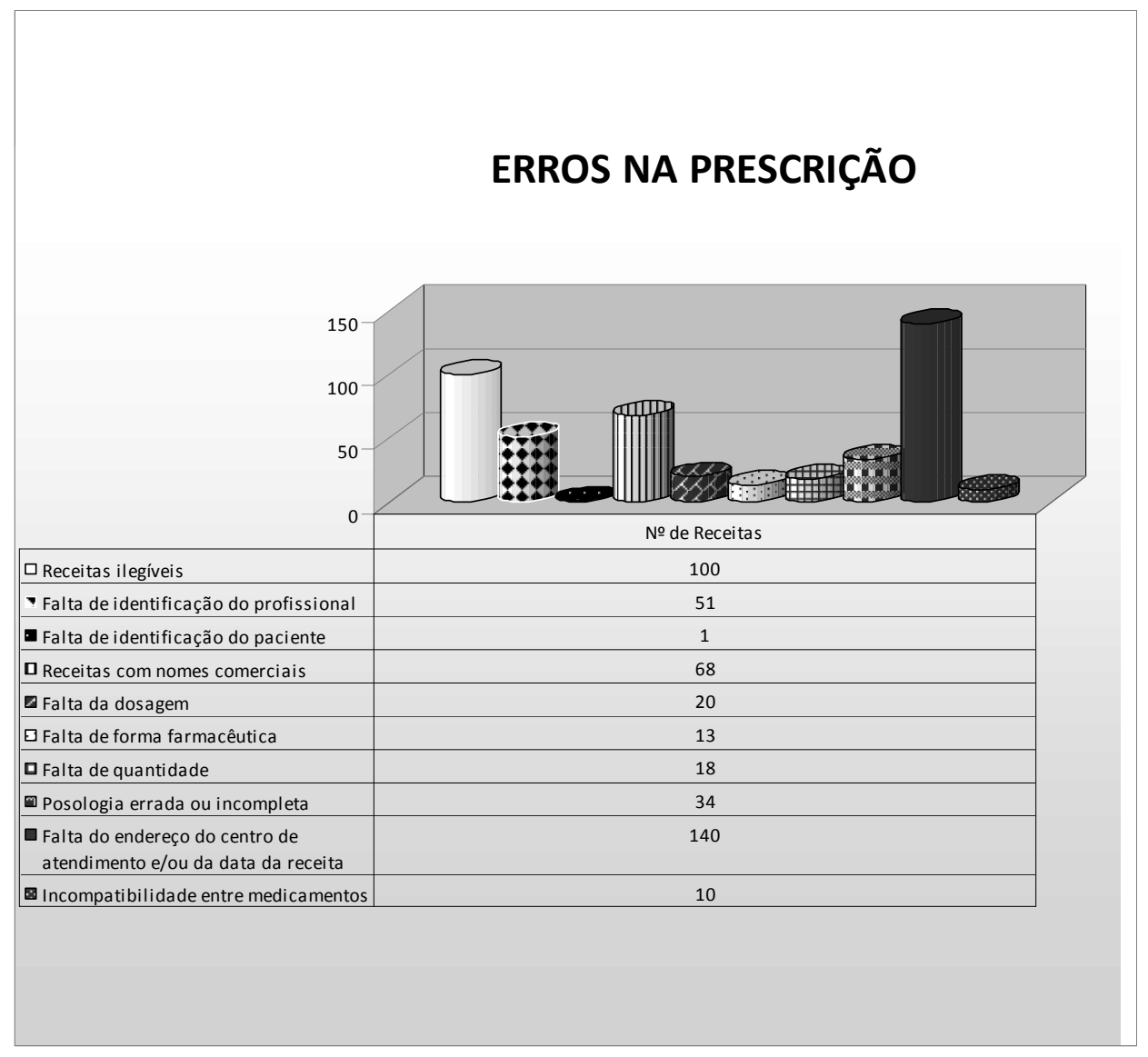

Fonte: os autores

\section{DISCUSSÃO}

As prescrições analisadas na Farmácia Escola da UNIFAL-MG, revelam que a falta de ilegibilidade ou dificuldade de leitura, impedem um rápido atendimento do farmacêutico e, mais ainda, dificulta o entendimento do paciente de sua própria receita. Neste trabalho, consideramos ilegibilidade quando foi impossível de entender o que estava escrito, sendo necessário consultar a opinião de outros 
farmacêuticos além do farmacêutico envolvido no momento da dispensação.

Assim quando não se consegue entender o que está escrito na receita, deve haver uma comunicação com o profissional prescritor para solucionar qualquer dúvida. A interação com o profissional prescritor não ocorre na maioria das drogarias possivelmente por esses motivos: ausência de dados do profissional, receio desta interação ou mesmo pelo tempo gasto nesta comunicação. Observamos que a ausência do endereço do profissional ou a falta de identificação deste dificultam a interação do farmacêutico durante a dispensação, obrigando o farmacêutico a deduzir qual o medicamento deve ser dispensado. A ilegibilidade ou a falta da identificação correta da receita pode levar a dispensação errada de medicamento. A dispensação de medicamentos prescritos para populações especiais como criança e idosos além da necessidade de identificação deveria incluir a idade ou data de nascimento, para que estes possam ser identificados corretamente no momento da dispensação; essa simples informação já seria suficiente para evitar erros que podem causar sérias consequências, uma vez que a dose e forma de uso devem estar adequadas a essa população.

A Organização Mundial da Saúde (OMS) sugere a utilização do nome genérico (denominação comum) em todas as prescrições para facilitar o ensino e a informação. A não utilização da denominação genérica nas prescrições pode provocar dificuldades para o paciente na identificação do medicamento em função da confusão entre nomes comerciais e genéricos, além de promover a compra de medicamentos mais caros, ainda havendo alternativas de preços mais acessíveis (SILVA, 2004).

A prescrição que utiliza a denominação comum merece destaque, pois está em acordo com a Lei dos Genéricos, que determina que no âmbito do SUS todas as receitas devem utilizar a Denominação Comum Brasileira (DCB) ou na ausência desta, a Denominação Comum Internacional (DCI). No entanto nesta pesquisa muitas receitas apresentavam nomes comerciais dos medicamentos. Embora em consultórios ou em clínicas particulares não seja obrigatório o uso do DCB nas receitas. Observou-se que o uso de nomes comerciais é relativamente grande e estima-se que esta porcentagem deva ser maior uma vez que entre as prescrições analisadas havia um grande número proveniente de pacientes do SUS, que adota na maioria das vezes DCB.

Observamos, também, a presença de informações que poderiam deixar dúvidas aos profissionais na farmácia, tais como: o uso de abreviações, ausências de dados sobre a via de administração e dose da apresentação ou informações adicionais necessárias, mas não presentes. Constatamos, ainda que das receitas obtidas na Farmácia Escola algumas apresentaram posologia errada ou incompleta, obrigando o farmacêutico a interagir com o 
profissional prescritor e/ou pesquisar na literatura científica para atender o paciente, gerando uma considerável demora na dispensação do medicamento.

As prescrições devem conter a dose de apresentação do medicamento, para que ele seja solicitado à farmácia, e, também a dose prescrita, para que o farmacêutico, ou mesmo, o atendente de farmácia, saiba a quantidade exata que deve se administrar ao paciente. Verificou-se que, embora seja um erro grave, algumas prescrições não apresentavam dosagem ou não apresentavam a forma farmacêutica, dificultando a dispensação de medicamento, uma vez que alguns medicamentos apresentam mais de uma forma farmacêutica; e além disso observamos que algumas prescrições apresentavam a falta de quantidade ou duração do tratamento.

Um dos fatores que mais causam indignação é a ausência de comprometimento do profissional prescritor com a compreensão correta do paciente sobre o modo de utilizar o medicamento, desta forma encontramos algumas prescrições com a frase "uso conforme a orientação", transferindo para o

\section{CONCLUSÃO}

A prescrição é uma importante fonte de informações para o paciente e para o farmacêutico. A falta de padronização e, as vezes, a falta de atenção ao prescrever dificultam o uso racional de medicamentos, pois o paciente fica impossibilitado de compreender o próprio tratamento a que está paciente a responsabilidade de lembrar-se do que foi orientado no momento da consulta, mesmo sob o risco de uma eventual confusão por parte do paciente. Esse tipo de prescrição impossibilita qualquer agente de saúde prever qual a orientação que o prescritor informou ao usuário.

Esses resultados demonstram que os profissionais habilitados a prescrever medicamentos precisam ser conscientizados de que a qualidade das prescrições influencia diretamente a qualidade do tratamento. A prescrição não deve ser realizada com pressa, falta de atenção e descaso; ela representa o resultado final da consulta médica ou odontológica e o início do tratamento farmacológico deste paciente.

A incompatibilidade entre os medicamentos também foi encontrada, esse erro passaria despercebido por um atendente de farmácia pois, apenas o farmacêutico tem habilidade técnica para detectar esse tipo de erro. Apesar de ocorrer em menor proporção, é um agravante, pois o efeito entre os medicamentos pode gerar resultados indesejáveis para o paciente.

sendo submetido e, o farmacêutico, fica limitado na prática da dispensação farmacêutica diante de informações erradas e/ou incompletas.

Assim para se evitar erros devidos à redação da prescrição médica, estudos apontam medidas que têm sido tomadas, 
como: padronização de processos, uso da prescrição eletrônica, aprimoramento e ênfase à educação dos profissionais que prescrevem, e expansão da ação dos farmacêuticos clínicos para melhor integração da equipe de saúde. Freire et al., 2004 afirma que a implantação da prescrição eletrônica é uma das tentativas mais sérias e mais efetivas para a precaução dos erros na medicação.

Desta forma, para diminuir os problemas em relação à prescrição poderia ser implantando as prescrições eletrônicas, as quais se utiliza o sistema computadorizado, de digitação, segundo um modelo de disposição de dados. Esse tipo de prescrição oferece um aumento da segurança, já que elimina possíveis problemas, na leitura e na compreensão, ocasionadas pela letra ilegível, possibilitando que a falha seja reparada no

\section{REFERÊNCIAS}

BRASIL. Agência Nacional de Vigilância Sanitária - Lei $n^{0} 5.991$, de 17 de novembro de 1973. Dispõe sobre o controle sanitário do comércio de drogas, medicamentos, insumos farmacêuticos e correlatos, e dá outras providências. Diário oficial da República Federativa do Brasil. Brasília, DF, 21 dez. 1973. Disponível em: <www.anvisa.gov.br> .Acesso em 14 jul. 2009.

BROWN, T. A. Reviewing and dispensing prescription and medication orders. In: Comprehensive Pharmacy Review (L. Shargel, A. H. Mutnick, P. F. Souney, L. N. Swanson \& L. H. Block, eds.). Baltimore: Williams \& Wilkins. p. 428-435, 1997. instante da digitação reduzindo significativamente a frequência dos sérios erros na medicação (FREIRE et al.; 2004).

Este trabalho demonstra a má qualidade das prescrições de medicamentos, indicando um despreparo ou mesmo indiferença dos profissionais prescritores para com o usuário de medicamentos. Faz-se necessário sensibilizar os profissionais de saúde à importância da qualidade da prescrição, para dispensação e administração de medicamentos e promoção do uso racional, proporcionando ao paciente maior eficácia e segurança do seu tratamento farmacológico.

\section{AGRADECIMENTO}

A Farmácia-Escola da UNIFAL-MG e ao Centro de Farmacovigilância da UNIFALMG (CEFAL) pelo subsídio das receitas.

COLOMBO, D.; SANTA-HELENA, E. T. AGOSTINHO, A. C.M.G.;DIDJURGEIT, J. S. M. A. Padrão de prescrição de medicamentos nas unidades de programa de saúde da família de Blumenau. Ver. Brás. Cienc. Farm. v.40,n.4, p.549-558, 2004.

FARIAS, A.D.; CARDOSO, M. A.A.; MEDEIROS, A.C.D.; BELÉM, L.F.; SIMÕES, M.O.S. Indicadores de prescrições médica na unidades básicas de saúde da família no município de Campinas Grande, PB. Rev. Bra. Epidemiol. v.10, n.2, p.149156, 2007.

FREIRE, C.C.; GIMENES, F.R.E.; CASSIANI, S.H.B. Análise da prescrição informatizada, em duas clínicas de um Hospital Universitário. Medicina, Ribeirão Preto, v. 37, p. 91-96, jan./jun. 2004. 
MEINERS M.M.M.A.; BERGSTEN-

MENDES G. Prescrição de medicamentos

para crianças hospitalizadas: como avaliar a

qualidade?. Rev. Assoc. Med. Bras.. v. 47,

n.4, dez. 2001.

PEPE, V.L.E.; CASTRO, C.G.S.O. A

interação entre prescritores, dispensadores e

pacientes: informação compartilhada como

possível benefício terapêutico. Caderno

Saúde Pública, v. 16, n. 3, p. 815-822, 2000.

RUPP, M. T.; DeYOUNG, M.;

SCHONDELMEYER, S. W. Prescribing

problems and pharmacist interventions in

community practice. Medical Care. v.30, p.

926-940, 1992.

SILVA, P.V. O uso de medicamentos na

atenção básica em Londrina, Pr. Londrina:

Universidade Estadual de Londrina.

[Dissertação de Mestrado]. 2004. 